\title{
Anatomia da valva atrioventricular esquerda: I. As cúspides
}

\author{
Antônio B. Prado FORTUNA*, Gilson BARRETO*, Armando Mâncio de CAMARGO**
}

RBCCV $44205-68$

FORTUNA, A. B. P.; BARRETO, G.; CAMARGO, A. M. - Anatomia da valva atrioventricular esquerda

I. As cúspides. Rev. Bras. Cir. Cardiovasc., 3(3): 201-209, 1988.

RESUMO: Os resultados de um estudo anatômico da valva atrioventricular esquerda, realizado em 30 coraçōes aparentemente normais, conservados em formalina, são comparados aos dados da literatura, tendo por objetivo adequar a nomenclatura e os conceitos anatómicos clássicos aos atuais, baseados na ultra-sonografia e na prática cirúrgica corrente. O presente artigo tratará apenas das cúspides, sendo que os demais elementos anatômicos dessa valva atrioventricular esquerda fazem parte de uma "unidade aortoventricular", onde distinguimos a cúspide anterior, em continuidade com o trigono intervalvar e o anel aórtico, e um grupo de cúspides posteriores, em relação com a parede posterior do ventrículo esquerdo. A cúspide anterior está separada do grupo de cúspides posteriores, tanto no polo valvar superior como no inferior, por uma estrutura anatômica bem definida: a "lâmina juncional", superior e inferior, respectivamente. As cúspides do grupo posterior são designadas pela ordem numeral ordinal, a partir da lâmina juncional superior. Esta nomenclatura tem por objetivo destacar a cúspide como unidade funcional da valva e facilitar as referências ecocardiográficas e cirúrgicas, particularmente nos casos em que mais de 3 cúspides posteriores sâo encontradas.

DESCRITORES: valvas cardiacas, cúspides, anatomia; valvas cardiacas, anatomia.

\section{INTRODUÇÃO}

O interesse pelo estudo anatômico da valva atrioventricular esquerda renova-se periodicamente, com o surgimento de novos métodos propedêuticos ou cirúrgicos, como, atualmente, vem ocorrendo com o emprego da ultra-sonografia no diagnóstico, e do laser no tratamento de determinados tipos de valvopatia.

O presente trabalho tem por objetivo rever os conceitos clássicos sobre a anatomia da valva atrioventricular esquerda e adequá-las às práticas clínica e cirúrgica correntes. Para isso, dados da literatura foram levantados e comparados aos resultados de um estudo anatômico realizado em corações humanos, conservados em formalina. Dada a extensão da matéria, o assunto será abordado em 5 artigos consecutivos, cobrindo, cada um deles separadamente, as diferentes partes que com- pōem a valva atrioventricular esquerda: I. As cúspides; II. As lâminas; III. As cordas tendíneas; IV. Os músculos papilares; V. O "anel" valvar.

\section{As cúspides}

As cúspides constituem os elementos dominantes da valva atrioventricular esquerda. A revisão da literatura mostra existir, entre os autores, dos clássicos aos contemporâneos, divergências conceituais importantes, como, por exemplo, sobre o númro das cúspides, a existência ou nāo de cúspides acessórias e, até mesmo, sobre a nomenclatura de cada uma das cúspides em particular.

O presente trabalho tem por objetivo rever a anatomia das cúspides da valva atrioventricular esquerda, realizando, para isso, além da revisão da literatura, um estu-

Trabalho realizado no Departamento de Anatomia do Instituto de Biologia da Universidade de Campinas. Campinas, SP, Brasil.

- Do Departamento de Cirurgia da Faculdade de Ciências Médicas da Universidade de Campinas.

* Do Departamento de Anatomia do Instituto de Biologia da Universidade de Campinas.

Recebido para publicação em 1: de dezembro de 1988.

Endereço para separtas: Antônio Prado Fortuna. Rua Barreto Leme, 1865. 13100 Campinas, SP, Brasil. 
FORTUNA, A. B. P.; BARRETO, G.; CAMARGO, A. M. - Anatomia da valva atrioventricular esquerda: I. As cúspides. Rev. Bras. Cir. Cardiovasc., 3(3): 201-209, 1988.

do em peças anatômicas, orientado por conhecimentos atuais obtidos pela ultra-sonografia e pela experiência adquirida com a visão direta dessa valva, durante a cirurgia $^{12}$

\section{MATERIAL E MÉTODOS}

Foram estudados 30 coraçōes adultos, aparentemente normais, pesando entre 240 e 320 ( $285 \pm 29)$ gramos, sem evidência macroscópica de valvopatia, cardiomiopatia ou aterosclerose coronária. As peças, todas pertencentes à coleção didática do Departamento de Anatomia do Instituto de Biologia da Universidade Estadual de Campinas - UNICAMP - estavam fixados em formalina.

A valva atrioventricular esquerda foi exposta por ampla abertura do ventrículo esquerdo, iniciada desde a parede posterior do átrio correspondente, seccionandose ao meio a segunda cúspide posterior e prosseguindo na mesma direção até o ápex ventricular, inclusive. As bordas as incisão foram mantidas separadas por meio de grampos fixadores, desfazendo-se ao máximo a concavidade natural da peça fixada (Figura 1).

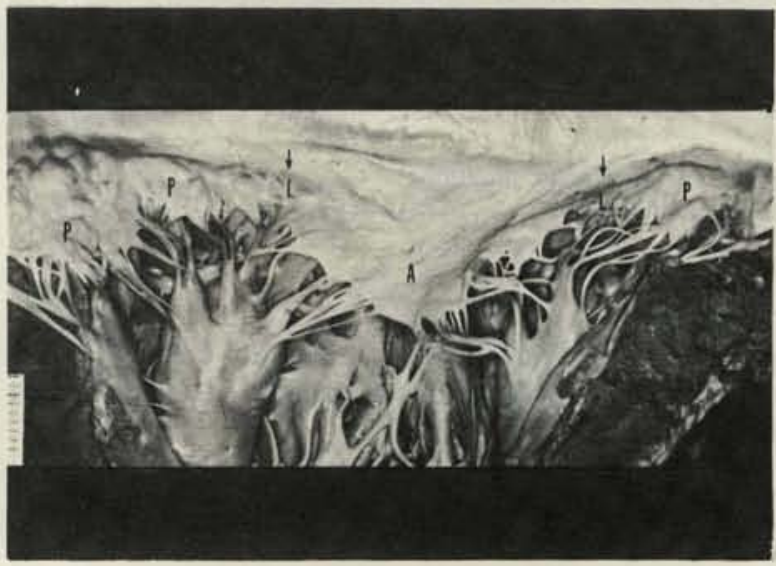

Fig. 1 - Valva atrioventricular esquerda: A) cuspide anterior; P) grupo posterior; L) lámina juncional superior e inferior.

As incisuras ou fendas encontradas na borda livre do manguito valvar foram consideradas como pontos de separação entre as cúspides. Particular atenção foi dada à regiāo das fendas que separam a cúspide, identificada como a "anterior", e o que designamos como "grupo de cúspides posteriores", procurando-se identificar, nessa regiāo, o que foi descrito pelos clássicos como "cúspides acessórias" 8. 13. 23. 27 e, mais recentemente, como "comissuras" 26.

Utilizando-se de um paquímetro, com subdivisōes de 0,1 centímetro, as seguintes medidas foram realizadas sobre as cúspides: 1) a altura, medida da base de implantação atrial ao ápice da borda livre; 2) a extensão, ou base, medida ao longo da linha de implantação atrial; a altura da "área nodosa" (zona áspera) e da "área lisa" (zona clara), identificadas, respectivamente, pelos métodos da transiluminação e da palpação.

\section{RESULTADOS}

Os dados referentes ao número de cúspides, considerado no presente trabalho, constam da Tabela 1.

TABELA 1

NÚMERO DE CÚSPIDES

\begin{tabular}{|c|c|c|}
\hline Cúspide & $N$ & $\%$ \\
\hline \multicolumn{3}{|l|}{ Anterior: } \\
\hline Uma cúspide & 30 & 100 \\
\hline \multicolumn{3}{|l|}{ Grupo posterior: } \\
\hline Duas cúspides & 1 & 3 \\
\hline Três cúspides & 27 & 90 \\
\hline Quatro cúspides & 2 & 7 \\
\hline
\end{tabular}

Os valores, em centímetros, encontrados para altura e extensão da cúspide anterior são apresentados na Tabela 2. Os valores da altura das áreas nodosa e lisa, nessa mesma cúspide, são apresentados na Tabela 3. Igualmente, os valores para o "grupo das cúspides posteriores" são apresentados nas Tabelas 4 e 5, respectivamente. Para os valores referentes à "altura" das cúspides posteriores e de suas respectivas áreas nodosa $e$ lisa, foram considerados os encontrados na segunda cúspide, por ter sido esta a cúspide mais expressiva do grupo posterior. Para o valor da "extensão" desse grupo, foi considerado o total da mediaa das bases de todas as cúspides posteriores, independente do número das mesmas.

TABELA 2

CUSSPIDE ANTERIOR - ALTURA E EXTENSÄO

\begin{tabular}{cc}
\hline Altura $(\mathrm{cm})$ & Extensão $(\mathrm{cm})$ \\
\hline $2,03 \pm 0,30(1,37-2,64)$ & $2,82 \pm 0,40(2,20-4,14)$ \\
\hline
\end{tabular}

TABELA 3

CÚSPIDE ANTERIOR - ALTURA AREAS NODOSA E LISA

\begin{tabular}{ccc}
\hline Area Nodosa $(\mathrm{cm})$ & Area Lisa $(\mathrm{cm})$ & $N /$ \\
\hline $0,83 \pm 0,14(0,53-1,10)$ & $1,20 \pm 0,24(0,73-1,83)$ & 0,69 \\
\hline
\end{tabular}


TABELA 4

GRUPO POSTERIOR - ALTURA E EXTENSÃO

\begin{tabular}{cc}
\hline Altura $(\mathrm{cm})$ & Extensão $(\mathrm{cm})$ \\
\hline $1,34 \pm 0,20(0,90-1,70)$ & $4,11 \pm 0,38(1,77-6,48)$ \\
\hline
\end{tabular}

TABELA 5

GRUPO POSTERIOR - ALTURA AREAS NODOSA E LISA

\begin{tabular}{ccc}
\hline Area Nodosa & Area Lisa & $N L$ \\
\hline $0,73 \pm 0,20(0,50-1,31)$ & $0,61 \pm 0,10(0,20-0,75)$ & 1,20
\end{tabular}

\section{COMENTÁRIOS}

\section{Nomenclatura anatómica}

A primeira dificuldade encontrada por aqueles que, entre nós, se propōem a estudar a valva atrioventricular esquerda, passa pela semântica: qual seria o termo correto: valva ou válvula? cúspide, lacínia ou folheto?

A consulta a 2 dicionaristas da língua portuguesa, mais citados atualmente, Caldas Aulete ${ }^{~ e ~ A u r e ́ l i o ~}{ }^{11}$, além de Paciornik ${ }^{22}$, dicionarista de termos médicos, nos trazem as seguintes definiçōes:

Valva - Segundo Aulete, é a "... peça ou cada uma das peças que consta a concha dos mariscos....". Para Aurélio é "... a parte destacável de um órgão cavitário..." (Botan.) e, segundo Paciornik, “... o conjunto de estruturas membranosas (válvulas) de um conduto ou passagem, que impedem o refluxo de seu conteúdo..."

Válvula - Segundo Aulete, significa "... membrana ou dobra membranosa que existe nos vasos sangüineos...". Para Aurélio, é "... uma pequena valva...", com o que concorda Paciornik, que define esse termo como "... diminutivo de valva...".

Folheto - Tanto para Aulete como para Aurélio, este termo significa "... livro de poucas folhas em brochura..." Paciornik não faz referência ao mesmo, como termo médico.

Lacínia - Para Aulete, significa "... lâmina, segmento..." (Anat.). Para Aurélio, "... o segmento de qualquer órgão foliáceo, profundo, estreito e agudo no ápice..." (Botan.). Paciornik não faz referência, também, a este termo.

Cúspide - Segundo Aulete, significa "... aṕice, extremidade aguda...". Para Aurélio, “... uma extremidade aguda, vértice, ... a porção afilada que terminam certos vegetais ... a saliência dos dentes...". Paciornik não faz constar essa palavra, em seu dicionário de termos médicos.
Pelo visto, a dificuldade em se encontrar o termo anatômico correto, para designar as cúspides da valva atrioventricular esquerda, tem-nos levado ao uso de termos, alguns inapropriados. A ausência de padronização dessa nomencltura pode ser sentida, desde os anatomistas clássicos, aos contemporâneos. Assim, por exemplo, BOURGERY ${ }^{3}$ refere-se às cúspides como a "direita" e a "esquerda", enquanto GRAY ${ }^{13}$ prefere os termos "ventral" e "dorsal"; POIRIER \& NICOLAS ${ }^{23}$, "interna" e "externa"; CRUVEILHER \& SÉE ${ }^{8}$, "maior" e "menor" e, mais recentemente, $\mathrm{CHIECHI}$ et alii ", CARPENTIER et alii ${ }^{5}$, WANSLEY ${ }^{30}$, "aórtica" e "mural"; BAILEY ${ }^{2}$, "septal" e "mural", e entre nós, MORAES ${ }^{20}$, "aórtica" e "posterior".

A nomenclatura recomendada pela Nomina Anatômica ${ }^{21}$, constitui a alternativa válida para os que buscam a padronização da terminologia anatômica. De acordo com esta referência, utilizaremos, no presente trabalho. os termos "valva atrioventricular esquerda", "cúspides anterior e posterior", procurando evitar termos ou expressões menos precisas, mesmo as já consagradas pelo uso, como: "o folheto aórtico da válvula mitral", ou "a lacínia mural ou externa da mitral".

Convém lembrar que os termos "anterior" e "posterior", referem-se à cúspides da valva atrioventricular esquerda no coraçāo in situ, do indivíduo na posiçāo anatômica, isto é, em pé, e nâo no de paciente em decúbito dorsal na mesa de cirurgia, ou em decúbito semilateral durante um exame ultra-sônico do coração.

\section{Número de cúspides}

Classicamente, a valva atrioventricular esquerda tem sido descrita como sendo formada por 2 cúspides, uma anterior e outra posterior, opostas uma à outra, lembrando a forma e a disposição de uma mitra, o que Ihe deu o nome de "valva mitral", termo este, segundo TANDLER ${ }^{27}$, atribuido a VESALE (1514-1564). GRAY ${ }^{13}$ preferia o termo "válvula bicúspide" (válvula bicuspidalis ), embora reconhecesse, entre ambas, a ocorrência de "cúspides acessórias".

Muitos autores, contrariando o conceito de valva bicúspide, descreveram a valva atrioventricular esquerda como sendo formada por 4 cúspides. Entre esses, distinguimos BOURGERY ${ }^{3}$, CRUVEILHIER \& SEE $^{8}$, CUNNINGHAN ${ }^{4}$, TESTU ${ }^{28} \mathrm{e}$, mais recentemente, YACOUB $^{31}$. Outros, como POIRIER \& NICOLAS ${ }^{23}$, TANDLER $^{27}$, HÁRKEN et alii ${ }^{14}$, RUSTED et alii ${ }^{26}, \mathrm{CHIECHI}$ et alii $^{7}$, MORAES ${ }^{20}$ DAVILA \& PALMER ${ }^{9}$, enfatizam que o achado de mais de 2 cúspides deva ser interpretado como ocasional. Mais recentemente, RANGANATHAN et alii ${ }^{24}$, baseados na distribuição das inserçōes cordais nas cúspides, estabeleceram o critério da ocorrência de 2 cúspides, a anterior e posterior, separadas. ambas, por uma "área comissural". 
Nossos achados correspondem mais à descriçāo inicial de $\operatorname{HENLE}^{15}$, que considera a valva atrioventricular esquerda como sendo constituida por uma faixa contínua de tecido valvar, apresentando uma borda fixa ao óstio atrioventricular, em continuidade com o anel aórtico, enquanto o outro, livre na cavidade ventricular, exceto pelas inserçōes cordais, apresenta, a intervalos regulares, um número variável de incisuras ou fendas. A frequeência e a profundidade dessas fendas é que determinam o número e a forma das cúspides. $\mathrm{A}$ análise cuidadosa dessas incisuras permite reconhecer que as mesmas não alcançam, em nenhum ponto, a borda fixa do manguito valvar, evidenciando-se, dessa forma, a continuidade do tecido valvar em toda sua extensão. O conceito de continuidade anatômica entre as cúspides, inicialmente esposado por $\operatorname{HENLE}^{15}$, foi aceito, também, por outros anatomistas clássicos, como POIRIER \& NICOLAS ${ }^{23}$, TANDLER $^{27}$, TESTU $^{28} \mathrm{e}$, mais recentemente, por $\mathrm{CHIECHI}$ et alii ${ }^{7}$.

Em nosso material, identificamos, em todas as peças, a ocorrência de uma cúspide anterior, dominante, e de um "grupo" de cúspides posteriores, que variou em número entre $2(2 / 30)$ e 4 cúspides $(1 / 30)$, tendo predominado, entretanto, o número de $3(27 / 30)$ (Tabela 1).

Não reconhecemos a ocorrência de "cúspides acessórias", mas constatamos, em cada pólo valvar, na área de junção da cúspide anterior com o grupo de cúspides posteriores, a ocorrência de uma curta faixa retangular de tecido valvar, a que chamamos de "lâmina juncional" superior e inferior, respectivamente. Essas estruturas correspondem, em parte, ás que foram descritas por $\mathrm{CHIECHI}$ et alii ${ }^{7}$ com o nome de "tecido juncional" e, mais recentemente, por RANGANATHAN et ali ${ }^{24}$ como "áreas comissurais".

\section{A cúspide anterior}

3.1 Aspecto Geral - A cúspide anterior, única, de forma trapezoídal, fixa à raiz aórtica, de onde se desprende como uma cortina na cavidade ventricular esquerda, separando a via de entrada da via de saída desse ventrículo. Apresenta 2 faces: a atrial e a ventricular; 1 base; 2 bordas laterais; e 1 ápice.

No aspecto atrial, a cúspide anterior apresenta-se lisa em toda extensão. Sua base está representada por um sulco semicircular bem definido, de concavidade voltada para dentro, resultante da reflexāo do endocárdio atrial sobre a cúspide. Duas pequenas depressōes ou fóveas, vistas na parede interna do átrio, logo acima do plano valvar, foram descritas por ZIMMERMAN ${ }^{32} \mathrm{CO}-$ mo pontos e referência anatômica às "comissuras". Essas fóveas resultam das inserçōes laterais da cúspide anterior nos trígonos fibrosos. A fóvea superior está em relação com a origem do ramo circunflexo da artéria coronária esquerda, e a fóvea inferior, com a porção membranosa do septo interventricular e o feixe de His.

Vista pelo aspecto ventricular, a base da cúspide anterior se continua e se confunde com a base de uma outra estrutura fibrosa, densa, de forma triangular cujo vértice termina no anel aórtico, entre as inserçōes das válvulas semilunares aórticas esquerda e a posterior (não coronariana). Esta estrutura fibrosa constitui o "trígono intervalvar", descrito por HENLE ${ }^{15}$ e, mais recentemente, por DU PLESSIS \& MARCHAND ${ }^{10}$, sob o nome, menos apropriado, de "espaço intervalvar".

Segundo DU PLESSIS \& MARCHAND ${ }^{10}, 0$ "trígono intervalvar" não deve ser considerado como parte da valva atrioventricular esquerda, pois o mesmo se situa acima do plano correspondente á implantação da base da cúspide anterior, quando vista pelo átrio esquerdo. Do ponto de vista cirúrgico, este trigono intervalvar tem importância fundamental nas técnicas que levam em consideraçāo a secçāo do mesmo, como forma de ampliação do anel aórtico ${ }^{18}$. Ecocardiograficamente, o "trígono intervalvar" corresponde a importante referência anatômica, designada "separaçăo aórtico-mitral" ${ }^{25}$, ou "MAS".

Vista ainda pelo aspecto ventricular, além da continuidade de sua base com o trígono intervalvar, a fixação lateral da cúspide anterior nos "trígonos fibrosos", pode melhor ser apreciada. O trígono fibroso superior está localizado imediatamente abaixo do nadir da válvula aórtica esquerda, e o inferior, imediatamente abaixo do nadir da válvula aórtica posterior (não coronariana) ${ }^{17}$.

A evidente continuidade entre a valva atrioventricular esquerda e a aórtica levou McALPINE ${ }^{19}$ a considerar essas duas estruturas como pertencentes a uma "unidade aortoventricular", da qual fariam, também, parte todas as demais estruturas fibrosas centrais, como os trígonos superior e inferior e seus respectivos prolongamentos ou a fila coronária, o trígono intervalvar e a porção membranosa do septo interventricular.

RANGANATHAN et alii ${ }^{24}$ descrevem, na face ventricular da cúspide anterior, ao longo da porção apical e de sua borda livre, uma área de aspecto irregular onde se iserem as cordas intermediárias. Devido à inserção cordal, essa região é mais espessa, apresentando-se, por isso, opaca à transiluminação e áspera à palpação, no que constrasta com o restante da cúspide, que é translúcido e liso. A evidente diferença no aspecto físico dessas duas regiôes levou aqueles autores ${ }^{24}$ a chamarem à regiâo opaca e irregular de "zona áspera", e a lisa e translúcida, de "zona clara". Parece-nos mais apropriado, entretanto, chamá-las de "área nodosa" e "área lisa", respectivamente.

A "área nodosa" tem a forma de uma crescente, sendo mais alta na porção apical da cúspide, diminuindo gradativamente em direçāo das lâminas juncionais, onde termina. Nessa área, no seu aspecto atrial, CHIARUGI ${ }^{6}$ 
descreve com o nome de nódulos de Albini, pequenos pontos esbranquiçados, dispostos em fileiras e que correspondem a pontos de inserção terminal de cordas tendíneas, na face ventricular da cúspide. Esses nódulos sẩo mais evidentes na valva de recém-natos que na do adulto e correspondem às mesmas estruturas referidas por TITUS 29 como "nódulos albinos" (noduli albini). Estes nāo devem ser confundidos com o que tem sido descrito como as "excrecências de Lambl", que, segundo DAVILA \& PALMER ${ }^{9}$, são pequenos nódulos esbranquiçados, localizados na superfície de coaptação das cúspides, resultantes de deposição de fibrina em "lesões de uso" ou trauma do endocárdio atrial nessa região. No restante, além da área nodosa, a cúspide anterior está constituida pela "área lisa", livres de inserções cordais, embora seja atravessada pela continuidade de algumas cordas inseridas na área nodosa.

As bordas laterais e o ápice da cúspide anterior, nas peças anatômicas, flutuam livres na cavidade ventricular esquerda e se prestam à inserção das cordas tendíneas do tipo marginal.

3.2 Dimensões - Os valores das medidas referentes a altura e extensão da cúspide anterior, encontrados na literatura e os obtidos no presente estudo, não apresentados na Tabela 6.

Os valores na cúspide anterior, referentes às medidas da altura da "área nodosa", "área lisa" e de seu respectivo índice, são comparados na Tabela 7 com valores correspondentes encontrados por RANGANATHAN et alii ${ }^{24}$.

\section{Grupo de cúspides posteriores}

4.1 Aspecto geral - O número de cúspides que constitui, no presente estudo, o grupo posteror, variou entre 2 e 4 (Tabela 1). No conjunto, o grupo posterior assume a forma retangular, apresentando uma borda fixa ou base, uma borda livre, uma face atrial e uma face ventricular. Ao longo da sua borda livre, ocorre a presença de incisuras ou fendas incompletas, que delimitam e determinam o número de cúspides $O$ grupo de cúspides posteriores está separado da cúspide anterior a cada pólo valvar, pelas lâminas juncionais. A face atrial : apresenta-se lisa em quase toda sua extensão. Na face ventricular, pode-se distinguir, como na cúspide anterior, a presença da área nodosa, onde se inserem cordas intermediárias, e a área lisa, caracteristicamente, nas cúspides posteriores, pode receber, em sua porção mais basal, a inserção de um número variável de pequenas e curtas cordas tendineas, nascidas, em geral, diretamente da parede ventricular (cordas basais). As bordas livres das cúspides posteriores apresentam inserçōes para as cordas do tipo marginal. A borda fixa, ou base, vista pelo aspecto atrial, está representada pela dobra da reflexão do endocárdio atrial sobre as cúspides.

TABELA 6

CÚSPIDE ANTERIOR - ALTURA E EXTENSĀO

\begin{tabular}{lrrrrr}
\hline Autores & $N$ & & Altura $(\mathrm{cm})$ & Extensão (cm) \\
\hline Rusted* & $(50)$ & 2,20 & $(1,60-2,90)$ & - \\
Chiechi* $^{*}$ & $(105)$ & 2,15 & $(1,80-3,20)$ & 3,50 & $(2,40-4,50)$ \\
Du Plessis $^{*}$ & $(10)$ & 2,70 & $(2,20-3,00)$ & 3,50 & $(3,00-4,50)$ \\
Hilário* $_{\text {Ranganathan* }}^{*}$ & $(50)$ & 2,30 & $(1,60-3,00)$ & 3,75 & $(3,00-4,50)$ \\
Carpentier $_{\text {Presente Estudo }}$ & $(50)$ & 2,30 & $(1,80-3,50)$ & 3,25 & $(1,80-4,80)$ \\
& $(50)$ & 2,30 & & 3,20 & - \\
\hline
\end{tabular}

- Média dos valores encontrados para os sexos masculino e feminino.

TABELA 7

CUSSPIDE ANTERIOR - ALTURA DAS AREAS NODOSA E LISA

\begin{tabular}{llccccc}
\hline Autores & $N$ & \multicolumn{2}{c}{ Area Nodosa $(\mathrm{cm})$} & \multicolumn{2}{c}{ Area Lisa $(\mathrm{cm})$} & $N L$ \\
\hline Ranganathan & $(50)$ & 0,85 & $(0,50-1,40)$ & 1,30 & $(1,00-2,10)$ & 0,65 \\
Presente Estudo & $(30)$ & $0,83 \pm 0,14(0,53-1,10)$ & $1,20 \pm 0,24(0,73-1,83)$ & 0,69 \\
\hline
\end{tabular}

\footnotetext{
- Média dos valores encontrados para os sexos masculino e feminino.
} 
No plano subendocárdico, tem sido descrito um "anel fibroso", onde as cúspides posteriores tomariam inserçāo ${ }^{9}$. 17. 27. De acordo com ZIMMERMAN ${ }^{32}$, esse "anel", na realidade, seria uma estrutura circular incompleta em suas porçōes anterior e posterior. A porção anterior estaria representada pelo espaço intertrigonal, e a posterior, ausente, estaria em relação com a segunda cúspide posterior, que, por falta de um suporte fibroso nessa regiāo, tomaria inserção diretamente na junção atrioventricular, constituindo, assim, o que foi descrito por LIOTA ${ }^{17}$ como "ponto débil" da junção atrioventricular esquerda. Nessa região, a cúspide posterior coloca-se em relação muito próxima com a porção atrioventricular do ramo circunflexo da artéria coronária esquerda.

Durante a sistole ventricular, o orificio atrioventricular se veda pelo choque e oposição das áreas nodosas das cúspides anterior e posterior, estabelecendo-se. nesse momento, um ângulo bem definido que se situa na linha de separação entre a área nodosa (área de vedação) e a área lisa (área de contenção) das respectivas cúspides. $O$ deslocamento desse ângulo para fora dessa linha, em um segmento valvar ou, mais precisamente, em uma das cúspides, caracteriza o que tem sido descrito como "prolapso", ocorrendo com maior freqüência nas cúspides posteriores.

4.2 Dimensões - Para o "grupo de cúspides posteriores", a "altura" foi medida, sempre, na cúspide mais "alta" do grupo, que, em nosso material, correspondeu, sempre, à segunda cúspide posterior. A "extensão" foi medida ao longo das bases de todas as cúspides poste- riores, indepedentemente de seu número. Os valores das medidas referentes à altura e à extensão do grupo das cúspides posteriores, e à altura das respectivas áreas nodosa e lisa, encontrados no presente estudo, não comparados aos da literatura, nas Tabela 8 e 9 , respectivamente.

\section{Dados da literatura}

A revisão da literatura demonstra que, além de divergências quanto à nomenclatura, existe, também, entre os autores, divergências conceituais importantes, como, por exemplo, quanto aos critérios que definem os limites anatômicos entre as cúspides. A falta desse critério tem gerado interpretaçōes diferentes sobre o significado de uma mesma estrutura valvar, fazendo com que a mesma seja considerada, por alguns, como uma cúspide acessória e, por outros, como parte integrante da cúspide posterior. Controvérsia como essa, foi, em parte, resolvida por RANGANATHAN et alii ${ }^{24}$, ao proporem que se considere como cúspide posterior todas as cúspides localizadas entre as áreas comissurais. Nós não somente estamos de acordo com esse critério, mas acrescentamos que essas cúspides sejam agrupadas sob o nome genérico de "grupo posterior" e que cada uma delas, individualmente, seja designada por ordem numeral ordinal, a partir do pólo valvar superior, mais precisamente da lâmina juncional superior. A individualização de cada cúspide facilitaria a referência cirúrgica ou ecocardiográfica das mesmas, o que nos parece justificável, pela tendência atual em se considerarem alterações segmentares da valva atrioventricular esquerda.

TABELA 8

GRUPO POSTERIOR - ALTURA E EXTENSÃO

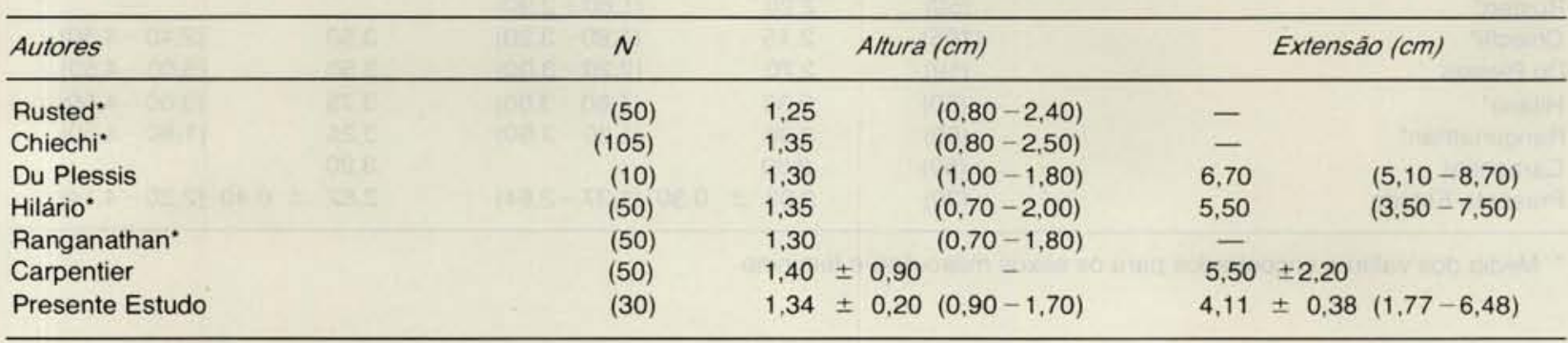

\footnotetext{
- Média dos valores encontrados para os sexos masculino e feminino.
}

TABELA 9

GRUPO POSTERIOR - ALTURA DAS AREAS NODOSA E LISA

\begin{tabular}{|c|c|c|c|c|c|}
\hline Autores & $N$ & Area Nodosa $(\mathrm{cm})$ & & Area Lisa $(\mathrm{cm})$ & $N L$ \\
\hline Ranganathan ${ }^{*}$ & (50) & $(0,30-1,40)$ & 0,50 & $(0,30-1,00)$ & 1,40 \\
\hline Presente Estudo & $(30)$ & $0,73 \pm 0,20(0,50-1,31)$ & $0.61 \pm$ & $\pm 0,10(0,20-0,75)$ & 1,20 \\
\hline
\end{tabular}


Quanto às dimensōes das cúspides, as medidas encontradas, no presente trabalho, estão bastante próximas às das verificadas na literatura (Tabelas 6,7 e 8 ). As diferenças constatadas por diversos autores poderiam ser explicadas por um fator racial e pela diferença na metodologia empregada. No presente estudo, a variação entre os sexos não foi considerada, pois acreditamos como CARPENTIER ${ }^{5}$ que o sexo não é fator relevante para estudos morfológicos, pois a diferença encontrada representa mais um fator relacionado ao tamanho do individuo que ao sexo propriamente dito. Quanto à metodologia, esta sim, pode ser responsável por algumas das diferehças encontradas. Em nossa série, o achado de valores menores, embora não significativos, referentes à extensão das cúspides, pode estar relacionado, além de fatores raciais, à acentuação da curvatura natural da cavidade ventricular esquerda das peças conservadas, utilizadas em nosso estudo.

\section{CONCLUSŌES}

1 Otermo "valva atrioventicular esquerda" deve ser preferido ao "válvula mitral".

2 Os elementos de vedação valvar devem ser designados "cúspides". Os termos "lacínias" ou "folhetos" são impróprios.

3 A valva atrioventricular esquerda é formada por uma manguito contínuo de tecido valvar, apresentando uma borda fixa em continuidade com a "unidade aortoventricular", e outra livre, onde a ocorrência de incisuras, ou fendas incompletas, detrminam o número de cúspides.

4 Reconhecemos a ocorrência de uma cúspide anterior, de forma trapezoidal, com a base voltada para cima. Vista pelo aspecto atrial, apresenta-se lisa, medindo em altura, isto é, de seu ápice à sua base, 2,03 $\pm 0,30(1,37-2,74) \mathrm{cm}$; e em extensão, medida ao longo de sua base, $2,82 \pm 0,40(2,20-4,14) \mathrm{cm}$. Vista pelo aspecto ventricular, sua base se confunde com 0 trigono intervalvar, uma estrutura fibrosa que a separa do anel aórtico (separação aórtico-mitral, "MAS"). Em sua porção apical, na face ventricular, observa-se uma "área nodosa", de forma crescente, que corresponde à região de inserção das cordas intermediárias. Deste ponto até a base, a cúspide anterior se continua na "área lisa". A área nodosa corresponde, durante a sistole ventricular, à área de oposição entre as cúspides e mede $0,83 \pm$ $0,14(0,53-1,10)$. A área lisa corresponde à área de contenção sistólica e mede $1,20 \pm 0,24(0,73-1,83)$. $\mathrm{O}$ índice área nodosa/área lisa (N/L) é $0,69 \mathrm{~cm}$.
$5 \mathrm{Na}$ junção entre a cúspide anterior e o grupo de cúspides posteriores, reconhecemos a ocorrência de uma estrutura bem definida, a lâmina juncional superior e a inferior. Duas pequenas depressões ou fóveas no endocárdio atrial, correspondendo à inserção trigonal da cúspide anterior, constituem pontos de referência atrial à localização das lâminas juncionais.

6 As cúspides posteriores são designadas por ordem numeral ordinal a partir da lâmina juncional superior. Essa nomenclatura tem por objetivo facilitar a referência cirúrgica ou ecocardiográfica das cúspides, particularmente no caso destas ocorrerem em número maior que 3.

7 Em nosso material, a presença de 3 cúspides posteriores ocorreu 27 vezes $(27 / 30)$, sendo a segunda, dominante. Em 2 ocasiōes (2/30), o grupo posterior esteve formado apenas pela primeira e segunda cúspide. não se diferenciando, nesses 2 casos, uma cúspide dominante. Em apenas uma ocasiāo (1/30), foi constatada a presença de uma quarta cúspide posterior. Neste caso, a segunda e a terceira foram, igualmente, dominantes.

8 Independentemente de seu número, as cúspides posteriores assumem, no conjunto, a forma retangular, cuja base comum se insere, no plano subendocárdico, nos prolongamentos posteriores dos trígonos fibrosos, a fila coronária. Esse suporte fibroso não é encontrado na região da segunda cúspide, constituindo o assim chamado "ponto débil" da junção atrioventricular esquerda, onde a referida cúspide se coloca em estreita relação com o ramo circunflexo da artéria coronária esquerda. A borda livre, onde se inserem as cordas marginais, apresenta um número variável de fendas ou incisuras, que delimitam as cúspides. $\mathrm{Na}$ face atrial, as cúspides se apresentam lisas e, na ventricular, pode-se reconhecer a área nodosa, onde se inserem as cordas intermediárias, e a área lisa, desprovida de inserção cordal, salvo para as curtas cordas basais, que se originam diretamente da parede vizinha do ventrículo esquerdo.

$9 \mathrm{~A}$ base comum às cúspides posteriores mediu 4,11 $\pm 0,38(1,37-6,48)$ em extensão. A altura do grupo posterior, verificada na segunda cúspide, em geral dominante, foi $1,34 \pm 0,20(0,90-1,70) \mathrm{cm}$. Nessa mesma cúspide, a área nodosa mediu $0,73 \pm 0,20(0,50-1,31)$ e a área lisa, $0,61 \pm 0,10(0,20-0,75)$, apresentando um indice $N / L$ igual a $1,20 \mathrm{~cm}$.

10 Os valores encontrados, no presente estudo, para as medidas das cúspides anterior, grupo posterior, e respectivas áreas nodosas e lisa, são bastante próximos aos da literatura. Pequenas diferenças foram interpretadas como decorrentes do fator racial, podendo, entretanto, em parte, serem devidas a variaçōes da metodologia empregada. 
FORTUNA, A. B. P.; BARRETO, G.; CAMARGO, A. M. - Anatomia da valva atrioventricular esquerda: I. As cúspides. Rev. Bras. Cir. Cardiovasc., 3(3): 201-209, 1988.

RBCCV $44205-68$

FORTUNA, A. B. P.; BARRETO, G.; CAMARGO, A. M. - Anatomy of the left atrio-ventricular valve: I. The cusps. Rev. Bras. Cir. Cardiovasc., 3(3): 201-209, 1988.

ABSTRACT: A review of the left atrio-ventricular valve anatomy is compared to a clinical oriented morphological study on 30 normal adult heart, preserved in formalin. The results will be presented in five separated sections: I. The cusps; II. The comissures; III. The chordae tendinae; IV. The papilary muscles; V. The valvar annulus. The present publication will deal with the cusps. The terms "left atrio-ventricular valve" and "cusps" are to be prefered to the more descriptive and traditional "mitral valve" and "leaflets". The anterior cusp, in continuity with the aortic annulus, and a group of posterior cusps, attached to the posterior left wall, are described. An ordinal numerical designation for the posterior cusps is proposed in order to facilitate ultrasonic and/or surgical references. The dimensions encountered for the cusps hight, including the "rough" and "clear" zones are superposed to those from others authors, but those refered to the extensions, are slightly small, probably due to more fixed, natural curvature of the fixed hearts.

DESCRIPTORS: heart valves, cusps, anatomy; heart valves, anatomy.

\section{REFERÊNCIAS BIBLIOGRÁFICAS}

1 AULETE, C. - Dicionário contemporâneo da lingua portuguesa. 3. ed. Rio de Janeiro, Delta, 1974.

2 BAILEY, C. P - Surgery of the heart. Filadelfia, Lea \& Febiger, 1955.

3 BOURGERY, J. M. - Traite complet de lanatomie de I'homme comprenant la medicine operatoire. Paris, Delaunay, 1835

4 BRASH, J. C. - Cunningham's textbook of anatomy. 9. ed. Londres, Oxford University Press, 1951.

5 CARPENTIER, A.; GUERINON, J.; DELOCHE, A.; FABIANI, J. N.; RELLAND, J. - Pathology of the mitral valve, In: KALMANSON, D. ed. The mitral valve: a pluridisciplinary approach. Londres, E. Ernold, 1976.

6 CHIARUGI, G. - Instituzioni di anatomia delloumo. 4. ed. Milano, Societá Editrice Libreria, 1936.

7 CHIEGHI, M. A.; LESS, W. W.; THOMPSON, R. - Funtional anatomy of normal mitral valve. J. Thorac, Surg., 32(3): 378-398, 1956.

8 CRUVEILHER, J. \& SÉE, M. - Traité d'anatomie descriptive.5. ed. Paris, P. Asselin, 1887. Tomo 3.

9 DAVILA, J. C. \& PALMER, T. E. - The mitral valve: anatomy and pathology for surgeon. Arch. Surg., 84(1): 38-62, 1962.

10 DU PLESSIS, L. A.; MARCHAND, P. - The anatomy of the mitral valve and its associeted structures. Thorax, 19(3): 221-222, 1964.

11. FERREIRA, A. B. H. - Novo dicionário da lingua portuguesa, Rio de Janeiro, Nova Fronteira, aspecto valvar. Campinas, 1983 (Tese-Faculdade de
Ciências Médicas, Universidade Estadual de Campinas).

13 GRAY, H. - Anatomia. WARNICK, R. \& WILLAISN, P. L. eds. Trad. Port.: SOUZA, O. M. et alii (35. ed.) Rio de Janeiro, Guanabara - Koogan, 1979.

14 HARKEN, D. E.; ELLIS, L. B.; DEXTER, L.; FERRAND. R. E.; DICKSON, J. F. - The responsibility of the physician in the selection of patients with mitral stenosis for surgical treatment. Circulation, 5(3): 349-362, 1952.

15 HENLE, J. - Jandbuch der systematischen Anatomie des menschen. Braunschweig, F. Vieweg, 1987.

16 HILÁRIO, J. - Contribuiçâo à cirurgia da válvula mitral. Rio de Janeiro, Casa Publicadora Batista, 1955. (Tese de doutorado, Faculdade Nacional de Medicina. Universidade do Brasil).

17 LOITA, D. - Anatomia cirurgica. In: LIOTA, D. ed. Técnicas em cirurgia cardiaca. Buenos Aires. Ed. Intermédica, 1980.

18 MANOUGUINA, S. \& SEYBOLD-EPTING, W. - Patch enlargement of aortic valve ring by extending the aortic incision into the anterior mitral leaflet. J. Thorac. Cardiovasc. Surg., 78(3): 402-412, 1979.

19 MCALPINE, W. A. - Heart and coronary arteries: an anatomical attas for clinical diagnosis, radiological investigation, and surgical treatment. New York, SpringerVerlag. Heidelberg, 1975.

20 MORAES, I. N. - Contribuição para o conhecimento da valva mitral no coração humano, com observaçóes sobre a arquitetura das cúspides, pelo método das linhas de fenda. São Paulo, 1956 (Tese de doutorado, Faculdade de Medicina da Universidade de São Paulo).

21 NOMINA ANATOMICA, 5. ed., Rio de Janeiro, Medsi, (Tradução: Comissāo de Nomenclatura. Sociedade Brasileira de Anatomia), 1984. 
Bras. Cir. Cardiovasc., 3(3): 201-209, 1988.

PACIORNIK, R. - Dicionário Médico. 3. ed. Rio de janeiro, Guanabara Koogan, 1978.

23

POIRIER, P. \& NICOLAS, A. - Traité d'anatomie humaine. 3. ed. Paris, Masson, 1912. Tomo 2.

24 RANGANATHAN, N. LAM, J. H. C, WIGLE, E. D.; SILVER, M. D. - Morphology of human mitral valve: II. The valves leaflets. Circulation, 41(3): 459-467, 1970.

25 ROSENQUIST, G. C.: CLARK, E. B.; SWEENEY, L. J.; MCALLISTER, H. A. - The normal spectrum of mitral and aortic valve discontinuity. Circulation, 54(2): 298-301, 1976.

RUSTED, I. E.; SCHEIFLEY, C. H.; EDWARDS, J. E. Studies of the mitral valve: I. Anatomic features of the normal mitral valve and associated structures. Circulation, 6(6): 825-831, 1952.
27 TANDLER, J. - Anatomie des Herzens. In: Badeleben. Handbuch der Anatomie des Menschen. 8. ed. Jena. Verlag Von. Gustav Fischer, 1913. Tomo 3.

28 TESTU, L. - Traité d'anatomie humaine. Paris, G . Doin. 1929. Tomo 2.

29 TITUS, L. - Anatomy and pathology of the mitral valve. In:Surgery for acquired mitral valve disease. Filadelfia, W. B. Saunders Co. 1967.

30 WALMSLEY, R. - Anatomy of human mitral valve in adult cadaver and comparative anatomy of the valve. $\mathrm{Br}$. Heart J., 40(1): 351-366, 1978.

31 YACOUB, M. - Anatomy of the mitral valve cordae and cusps. In: KALMENSON, D. ed. - The mitral valve: a pluridisciplinary approach. Londres, E. Ernold, 1976.

32. ZIMMERMAN, J. - The funtional and surgical anatomy of the heart. Ann. Roy. Cool. Surg. Eng., 39(3): 348 . 1966. 